\title{
A novel autosomal recessive orthostatic hypotension syndrome: and other updates on recent autonomic research
}

\author{
Mitchell G. Miglis ${ }^{1} \cdot$ Srikanth Muppidi ${ }^{2}$
}

Received: 3 November 2018 / Accepted: 7 November 2018 / Published online: 10 November 2018

๑) Springer-Verlag GmbH Germany, part of Springer Nature 2018

Keywords Alpha-synucleinopathy · Skin biopsy · Orthostatic hypotension · Genetic autonomic disorders · Multiple system atrophy

\section{CYB561: a novel orthostatic hypotension syndrome}

The differential diagnosis of orthostatic hypotension $(\mathrm{OH})$ is broad, however once a neurogenic source is suspected, the differential narrows considerably. Genetic causes represent a rare but important form of $\mathrm{OH}$ that typically manifest early in childhood. The classic genetic syndrome of neurogenic orthostatic hypotension $(\mathrm{nOH})$ is dopamine $\beta$-hydroxylase $(\mathrm{D} \beta \mathrm{H})$ deficiency, in which this crucial enzyme cannot convert dopamine to norepinephrine, leading to sympathetic failure.

In the article "Mutations in CYB561 causing a novel orthostatic hypotension syndrome," published in the March issue of Circulation Research, van den Berg and colleagues describe a new genetic syndrome of sympathetic failure [4]. The authors discovered two pairs of siblings, one pair in the Netherlands and another pair evaluated at Vanderbilt University (Nashville, USA), who experienced severe lifethreatening orthostatic hypotension since infancy. All four patients had very low concentrations of plasma norepinephrine and epinephrine, as in $\mathrm{D} \beta \mathrm{H}$ deficiency. However, when plasma $\mathrm{D} \beta \mathrm{H}$ activity was measured, it was normal, and when the $\mathrm{D} \beta \mathrm{H}$ gene was sequenced, it was normal as well.

To determine the genetic origin of the disorder, whole exome sequencing in the 2 affected Dutch sisters and their relatives, and from the 2 affected American sisters was performed. Several shared homozygous regions in the siblings

Srikanth Muppidi

muppidis@stanford.edu

Stanford Medical Center, Palo Alto, CA, USA

2 Stanford Neurosciences Health Center, 213 Quarry Road, 2nd Floor, Palo Alto, CA 94304, USA were identified, and the authors selected variants concordant with autosomal recessive inheritance and ran them through bioinformatics and in silico analysis. Based on this analysis, CYB561 emerged as the candidate gene.

CYB561 is a transmembrane enzyme present in catecholamine and neuropeptide secretory vesicles in neuroendocrine tissues, and is thought to function as an electron carrier for ascorbic acid (vitamin C). D $\beta \mathrm{H}$ uses ascorbic acid as a cofactor to convert dopamine to norepinephrine. Without functional CYB561, ascorbic acid concentrations cannot be maintained in the vesicle and norepinephrine cannot be synthesized.

After identification of the CYB561 mutation, expression of CYB561 was investigated using RNA analysis. The CYB561 gene was expressed throughout the body but especially concentrated in the brain in the cortex and hippocampus. Thankfully, both D $\beta \mathrm{H}$ deficiency and CYB561 mutations are responsive to droxidopa (L-dihydroxyphenylserine), a drug that bypasses the defective pathway and is converted directly to norepinephrine.

In summary, this novel article details the exciting discovery of a new genetic condition of $\mathrm{nOH}$ that resembles $\mathrm{D} \beta \mathrm{H}$ deficiency with $\mathrm{nOH}$, recurrent hypoglycemia, and low norepinephrine levels. Moreover it highlights the role of vitamin $\mathrm{C}$ in catecholamine synthesis. It is tempting to speculate that vitamin $\mathrm{C}$ deficiency may contribute to neurogenic orthostatic hypotension in some adult patients. These results were highlighted by Dr. Ron Wevers, the senior author of the article, at the Groundbreaking Research Plenary Lecture during the 29th International Symposium on the Autonomc Nervous System in Newport Beach, California (October 2018). 


\section{Can skin biopsy help us diagnose multiple system atrophy?}

It is now well known that idiopathic Parkinson disease (PD), multiple system atrophy (MSA), dementia with Lewy bodies (DLB), and pure autonomic failure (PAF) all have one molecular feature in common: the pathological accumulation of the protein $\alpha$-synuclein ( $\alpha$-syn) in the central and peripheral nervous systems. These four disorders, while similar in many ways, are very different in others, with heterogenous phenotypes and variable prognoses, prompting scientists to question whether there might be distinct pathological variants of $\alpha$-syn deposition unique to each diagnosis.

Donadio and colleagues have attempted to better understand this question with their recent publication, "Skin $\alpha$-synuclein deposits differ in clinical variants of synucleinopathy: an in vivo study," published in the September issue of Scientific Reports [1]. In this study the authors enrolled 44 patients diagnosed with synucleinopathy, including 15 patients with IPD, 12 with probable DLB, 5 with PAF, and 12 with MSA (5 MSA-P and 7 MSA-C). The patients had similar periods of disease duration across diagnoses. Diagnoses were supported by ancillary testing which demonstrated cardiac postganglionic sympathetic denervation (123-I-MIBG), dopaminergic nigrostriatal abnormalities (123I-ioflupane-DatScan) or brainstem and cerebellum atrophy and/or the hot-cross bun sign (brain MRI). Ten age-matched healthy subjects were recruited as controls. Standard $3 \mathrm{~mm}$ punch skin biopsies were taken from the cervical $\mathrm{C} 7$ paravertebral area, proximal thigh $(15 \mathrm{~cm}$ above the patella) and distal leg $(10 \mathrm{~cm}$ above the lateral malleolus), as in the authors' prior publications. Two samples were taken in each skin site 3-4 cm away. Ten $\mu \mathrm{m}$ skin sections were double-immunostained with a panel of antibodies against $\alpha$-syn epitopes and the panneuronal marker protein gene product 9.5 (PGP). Different primary antibodies against normal or abnormal $\alpha$-syn and ubiquitin were double stained to characterize the abnormal $\alpha$-syn deposits.

P-syn staining showed the highest rate of positivity and specificity as it was found in most patients but in no controls. Interestingly, the authors found that abnormal $\alpha$-syn deposits were found in all patients except 4 with MSA-C, despite the fact that all of these patients had orthostatic hypotension. In addition, MSA showed abnormal aggregates only in somatosensory skin fibers, mainly those located in the subepidermal plexus, and with a different pattern of staining (dot-like, compared to the more homogenous staining of IPD, DLB and PAF). In contrast, the IPD, DLB and PAF demonstrated p-syn deposits in autonomic fibers and plexuses close to autonomic annexes. The authors also noted that the antibody against $\alpha$-syn phosphorylation at serine 129 demonstrated optimal sensitivity and specificity in disclosing Lewy neuritis in skin nerves. This finding has been reported in prior publications.

The limitations of this study include small sample sizes, with only 5 PAF patients. Larger cohort analyses will be necessary to confirm the authors' findings. Most interesting would be a prospective study in which the evolution of dermal $\alpha$-syn could be followed over time, including those with REM-sleep behavior disorder (RBD), now recognized as a form of prodromal $\alpha$-synucleinopathy. The authors reported that $15 \%$ of their IPD patients had RBD, though it is not clear in the methods section how the authors screened for RBD, which is difficult to confidently diagnose without video polysomnography. Assuming this percentage was accurate, this may have influenced the skin biopsy results; we know that RBD indicates more aggressive disease in patients with IPD, and the data is underpowered to perform subgroup analysis. This would also be interesting to analyze in future studies. Of note, other skin biopsy studies in patients with clinically isolated RBD have demonstrated that the majority demonstrate abnormal p-syn deposits in skin somatosensory fibers, as in the MSA patients in this study. It also not clear how orthostatic hypotension was measured, and if patients underwent autonomic reflex testing in a laboratory setting. Correlation with skin biopsy results would be an interesting variable to compare across diagnoses. Nonetheless, the finding that p-syn deposits in skin somatosensory fibers in MSA and not in other synucleinopathies is powerful, and may be serve as a useful biomarker to help distinguish to MSA from other synucleinopathies in prodromal patients.

\section{Nonpharmacological treatments for orthostatic hypotension: finally some evidence!}

Orthostatic hypotension $(\mathrm{OH})$ is one of the most common referrals seen in the autonomic clinic. Current consensus guidelines [2] encourage non-pharmacological treatment before initiating medications in patients with $\mathrm{OH}$. Classical non-pharmacological approaches include bolus water drinking $(500 \mathrm{ml})$, compression stockings, abdominal binder and physical countermaneuvers. While the benefit from these interventions has been evaluated in small groups of patients, not all measures were studied in the same patients and the relative efficacy of these measures remained unclear.

Newton and Frith aimed to clarify this question, recently publishing evidence in the journal Neurology on the relative efficacy of various nonpharmacological treatments in patients with $\mathrm{OH}$ [3]. They recruited 25 patients from their outpatient clinic with $\mathrm{OH}$ thought to be solely due to aging. All patients also had autonomic testing and other causes 
were excluded. All patients had supine and standing blood pressure measured at baseline and after drinking water bolus on visit one. Supine and standing BP was also measured after wearing an abdominal binder, compression stockings and preforming physical countermaneuvers on visit two. Patients completed the Orthostatic Hypotension Questionnaire to assess subjective improvement in orthostatic symptoms. The authors defined a response rate of $10 \mathrm{mmHg}$ increase in the standing systolic blood pressure (SBP) as the primary outcome measure.

Bolus water drinking was the most effective with $56 \%$ of patients having a $>10 \mathrm{mmHg}$ increase in SBP, followed by abdominal binder in $52 \%$ and physical countermaneuvers in $44 \%$. Compression stockings were least effective and only $32 \%$ of patients had a $10 \mathrm{mmHg}$ increase in standing SBP with this intervention. Interestingly, in spite of improvement in standing SBP with some of these interventions, none of these methods improved symptoms.

This study now provides some evidence to distinguish the nonpharmacological interventions we all recommend in the clinic, and favors bolus water drinking, abdominal binder and physical countermaneuvers over the value of compression stockings. However, it should be noted that these findings might not translate to all patients in the autonomic clinic. This study included patients with neurogenic $\mathrm{OH}$, however the authors did not describe how this was defined, or if the $\mathrm{OH}$ was symptomatic at baseline. This is relevant as it seems unusual for patients to experience a $10 \mathrm{mmHg}$ improvement in standing SBP and yet not report any clinically meaningful improvement in symptoms. It may be that patients only note improvement of symptoms with prolonged implementations of the above interventions. Finally, the authors note that there were no adverse effects; while we expect this, it would have been interesting to know if patients experienced any discomfort with these measures and the likelihood of following each intervention at home if it was recommended. In spite of these study limitations, this type of research study was overdue and helps autonomic physicians recommend appropriate non-pharmacological interventions.

Funding None.

\section{Compliance with ethical standards}

Conflict of interest All authors declare that they have no conflict of interest

\section{References}

1. Donadio V, Incensi A, El-Agnaf O et al (2018) Skin $\alpha$-synuclein deposits differ in clinical variants of synucleinopathy: an in vivo study. Sci Rep 8(1):14246. https://doi.org/10.1038/s41598-01832588-8

2. Freeman R, Wieling W, Axelrod FB et al (2011) Consensus statement on the definition of orthostatic hypotension, neurally mediated syncope and the postural tachycardia syndrome. Auton Neurosci 161(1):46-48. https://doi.org/10.1016/j.autneu.2011.02.004

3. Newton JL, Frith J (2018) The efficacy of nonpharmacologic intervention for orthostatic hypotension associated with aging. Neurology 91(7):e652-e656. https://doi.org/10.1212/WNL.00000 00000005994

4. van den Berg MP, Almomani R, Biaggioni I et al (2018) Mutations in CYB561 causing a novel orthostatic hypotension syndrome. Circ Res 122(6):846-854. https://doi.org/10.1161/CIRCRESAHA .117 .311949 